J. Lake Sci. (湖泊科学), 2014, 26(4): 593-599

http://www. jlakes. org. E-mail : jlakes@niglas.ac.cn

(C) 2014 by Journal of Lake Sciences

\title{
千岛湖表层沉积物中有机氯农药的残留特征及生态风险评价"
}

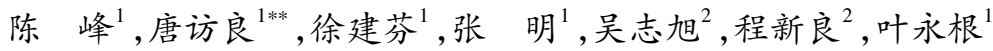 \\ $(1:$ 杭州市环境监测中心站,杭州 310007) \\ $(2$ : 淳安县环境监测站,杭州 311700$)$
}

摘 要: 对千岛湖表层沉积物中 21 种有机氯农药 $(\mathrm{OCPs})$ 的残留现状进行调查,明确 $\mathrm{HCHs}$ 与 DDTs 的组成特征及源解析, 并评估其生态风险. 结果表明, 千岛湖表层沉积物中共检出 12 种 OCPs, 各采样点位 OCPs 总量的浓度范围在 $0.43 \sim$ $12.70 \mathrm{ng} / \mathrm{g}$ 之间, 平均值为 $5.28 \pm 4.84 \mathrm{ng} / \mathrm{g}$, 处于低残留水平, 其中 DDTs 是主要的残留物, 街口、大坝前点位样品出现 OCPs 高残留. 工业 DDTs 的历史使用是千岛湖表层沉积物 DDTs 残留的主要来源, 仅街口点位样品存在林丹的污染并有 新的 DDTs 输人,应引起重视. 利用沉积物质量基准法、沉积物质量标准法分别对千岛湖表层沉积物中 OCPs 的生态风险 进行评价,结果表明部分点位样品中 $\mathrm{OCPs}$ 的残留现状对该区生物可能存在生态风险.

关键词: 有机氯农药;沉积物;千岛湖;残留特征;生态风险评价

\section{Residue characteristics and ecological risk assessment of organochlorine pesticide (OCPs) in the surface sediments of Lake Qiandao}

\author{
CHEN Feng ${ }^{1}$, TANG Fangliang ${ }^{1}$, XU Jianfen ${ }^{1}$, ZHANG Ming ${ }^{1}$, WU Zhixu ${ }^{2}$, CHENG Xinliang ${ }^{2} \&$ YE $^{2}$ \\ Yonggen $^{1}$ \\ (1: Hangzhou Environmental Monitoring Central Station, Hangzhou 310007, P. R. China) \\ (2: Chun' an Environmental Monitoring Station, Chun' an 311700, P. R. China)
}

Abstract: A survey was conducted on twenty-one kinds of organochlorine pesticide( OCPs) residue in the surface sediments of Lake Qiandao. The component characteristics and source apportionment of HCHs and DDTs were confirmed, and the ecological risk assessment was evaluated. The main results were as follows: twelve kinds of OCPs were detected, total concentrations of OCPs in the surface sediments of the studied area ranged from 0.43 to $12.70 \mathrm{ng} / \mathrm{g}$, with an average of $5.28 \pm 4.84 \mathrm{ng} / \mathrm{g}$, belonging to low residue level. DDTs were the main residues, and high OCPs residue level was found in Jiekou and Dabaqian. The application of industrial DDTs was the main source apportionment of DDTs residue in the surface sediments. Lindane and DDTs in the surface sediment of Jiekou were input recently, which should be paid more attention. In addition, the ecological risk of OCPs in the surface sediment of Lake Qiandao was assessed with the methods of sediment quality guidelines and sediment quality standards, and the results indicated that OCPs residue in some sampling sites may still have potential ecological risk on the investigated area.

Keywords: OCPs; sediment; Lake Qiandao; residue characteristics; ecological risk assessment

联合国环境规划署在 POPs 公约中首批列出 12 种最具有风险的持久性有机污染物:多氯二苯并二恶英、 多氯联苯、滴滴涕、狄氏剂、异狄氏剂、艾氏剂、氯丹、七氯、六氯苯、灭蚁灵、毒杀芬和多氯二苯并呋喃,其中 9 种 是有机氯农药 ${ }^{[1]}$. 作为一类广谱杀虫剂,有机氯农药曾被广泛用于农业和非农业生产中 ${ }^{[2]}$. 因为它既经济又有 效,在 1950s, DDTs 和 HCHs 等有机氯农药在中国曾被广泛使用,直到 1983 年才被限制生产和使用.

有机氯农药可通过喷酒、土施以及处理种子等途径进人农田环境 ${ }^{[3]}$. 当这类农药进人环境后, 一部分可

* 杭州市社会发展科研攻关项目 (20120433B05)、浙江省科技计划项目 (2013C37093) 和杭州市环境保护科研课题项 目 (2011003) 联合资助. 2013-08-10 收稿;2014-01-07 收修改稿. 陈峰(1983 ), 男,硕士研究生, 工程师; E-mail: chenfeng_1218@ hotmail. com.

** 通信作者;E-mail: tangfl001@163.com. 
以通过挥发进人大气环境散失; 另一部分可被植物吸收或穿过土壤的非饱和区运移到地下水; 也可随地表 径流进人地表水, 有机氯农药具有很强的疏水亲脂性, 进人水环境系统后, 大多数有机氯农药与悬浮颗粒物 质如矿物、生物碎屑和胶体等相结合并最终富集于表层沉积物中 ${ }^{[4]}$. 积蓄在沉积物中的有机氯农药一方面 通过再悬浮和解吸作用, 再次进人水体而造成二次污染; 另一方面, 水体中的有机氯农药被生物体摄人后不 易分解, 并沿着食物链富集放大, 从而危害人体健康 ${ }^{[5]}$.

千岛湖是 1959 年新安江水电站拦坝蓄水形成的人工湖, 是长三角地区重要的淡水水资源地和渔业生 产基地. 近年来,杭州市将启动千岛湖引水工程, 使千岛湖水环境质量受到前所未有的关注,一些学者对该 湖的营养盐负荷、叶绿素 $\mathrm{a}$ 及浮游动植物 ${ }^{[6-9]}$ 等进行了广泛研究, 但有关有机物污染方面的研究鲜见报道. 因此, 本文以千岛湖表层沉积物为研究对象, 通过对沉积物中 21 种有机氯农药 (OCPs) 的分析, 研究 OCPs 的残留水平与组成特征, 并探讨其可能来源与生态风险, 以期为千岛湖的有机污染控制提供基础数据.

\section{1 材料与方法}

\section{1 样品采集}

2012 年 12 月在千岛湖进行表层沉积物样品的采集, 选取千岛湖有代表性的区域共 7 个采样点 (图 1), $1 \sim 7$ 号采样点名称分别为街口、小金山、茅头尖、航头岛、三潭岛、西园库湾和大坝前, 其中街口为安徽上游 进千岛湖的人境断面, 流量约占总人湖流量的 $60 \%$, 茅头尖与航头岛为淳安县境内人湖的来水断面, 小金山 与三潭岛为千岛湖中心湖区断面, 西园库湾为城区库湾断面, 大坝前为出境断面. 表层沉积物样品利用抓斗 式采样器在每个断面采集 1 个表层沉积物样品. 样品采集后置于事先用丙酮清洗的广口棕色玻璃瓶中, 现 场用冰块保存, $24 \mathrm{~h}$ 内运回实验室于 $-20^{\circ} \mathrm{C}$ 冷冻保存备用.

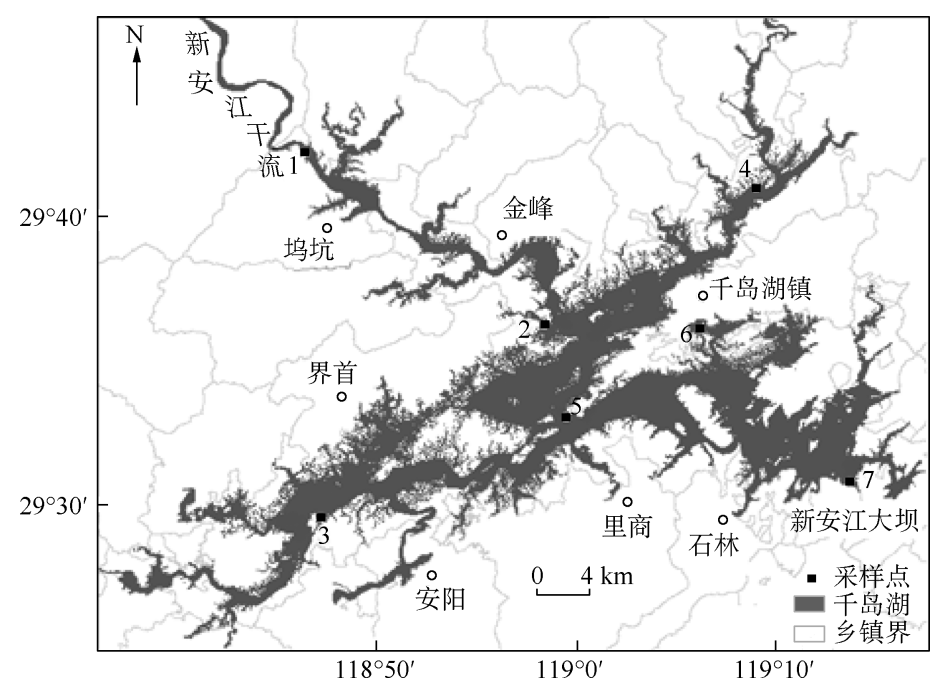

图 1 千岛湖采样点分布

Fig. 1 Distribution of sampling sites in Lake Qiandao

\section{2 标准样品与试剂}

包含 21 种 $\mathrm{OCPs}\left(\alpha-\mathrm{HCH}\right.$ 、六氯苯、 $\beta-\mathrm{HCH} 、 \gamma-\mathrm{HCH} 、 \delta-\mathrm{HCH}$ 、七氯、艾氏剂、异艾氏剂、环氧七氯、o, $\mathrm{p}^{\prime}-$ DDE、硫丹 I 、 p, p' -DDE、 $o, p^{\prime}$-DDD、狄氏剂、异狄氏剂、p, p' -DDD、 $o, p^{\prime}$-DDT、硫丹 II 、 p , p' -DDT、甲氧氯、 灭蚁灵) 的混合标准溶液为美国 o2si smart solutions 公司产品; 正已烷、丙酮均为农残级, 购自美国 TEDIA; 浓 硫酸为优级纯, 购自国药集团; 无水 $\mathrm{Na}_{2} \mathrm{SO}_{4}$ 为分析纯, 购自国药集团, 使用前于 $400^{\circ} \mathrm{C}$ 的马弗炉中活化 $6 \mathrm{~h}$. 空 白沉积物样品经冷冻干燥后研磨过䇥, 并在 $400^{\circ} \mathrm{C}$ 马弗炉中烘烤 $6 \mathrm{~h}$, 密封保存.

\section{3 样品预处理}

沉积物经冷冻干燥后研磨过 80 目篮, 使用 ASE 300 加速溶剂萃取仪 (Dionex, 美国) 萃取. 称取 $10 \mathrm{~g}$ 沉 
积物样品、 $5 \mathrm{~g}$ 中性氧化铝和 $2 \mathrm{~g}$ 铜片充分混合后加人 $34 \mathrm{ml}$ 萃取池中提取净化. 萃取条件为丙酮: 正己烷 (体积比为 $1: 1$ ), 加热温度为 $100^{\circ} \mathrm{C}$, 萃取压力 $1500 \mathrm{psi}$, 静态萃取循环 3 次, 溶剂快速冲洗样品体积比为 $60 \%$, 氮气吹扫收集提取液时间为 $60 \mathrm{~s}$. 样品提取液用 $2 \%$ 硫酸钠水溶液洗去丙酮, 用浓硫酸净化, 用 $2 \%$ 硫 酸钠水溶液洗到中性, 并经无水硫酸钠脱水,使用全自动氮吹浓缩仪( LabTech, 美国) 浓缩至约 $0.8 \mathrm{ml}$ 后, 用 正己烷定容 $1.0 \mathrm{ml}$, 待 $\mu \mathrm{ECD}$ 检测.

\section{4 检测与质控}

OCPs 的测定使用气相色谱仪 (Agilent 7890A). 采用的色谱条件为: 色谱柱: HP-5ms $60 \mathrm{~m} \times 0.25 \mathrm{~mm} \times$ $0.25 \mu \mathrm{m}$; 柱流速: 氮气 $1.2 \mathrm{ml} / \mathrm{min}$, 恒定; 进样口: 温度 $250^{\circ} \mathrm{C}$, 不分流进样; 柱室: 程序升温, 起始温度 $80^{\circ} \mathrm{C}$, 升温速率 $30^{\circ} \mathrm{C} / \mathrm{min}$ 到 $180^{\circ} \mathrm{C}$, 升温速率 $3^{\circ} \mathrm{C} / \mathrm{min}$ 到 $205^{\circ} \mathrm{C}$, 保持 $4 \mathrm{~min}$, 升温速率 $20^{\circ} \mathrm{C} / \mathrm{min}$ 到 $290^{\circ} \mathrm{C}$, 保持 $4 \mathrm{~min} ; \mu \mathrm{ECD}$ 检测器: 温度 $300^{\circ} \mathrm{C}$, 尾吹氮气流量: $60 \mathrm{ml} / \mathrm{min}$, 进样量: $1 \mu \mathrm{l}$.

质控手段主要为方法空白、样品平行、加标回收, 同时为了剔除假阳性, 对部分有质疑的检测结果重新 用三重四级杆气相色谱质谱联用仪 (GC-MS/MS 7000B) 进行分析确认. 方法检出限为 $0.01 \sim 0.17 \mathrm{ng} / \mathrm{g}$. 称 取 6 个 $10 \mathrm{~g}$ 空白沉积物样品, 分别加人 $1.0 \mathrm{ml} 500 \mu \mathrm{g} / \mathrm{L}$ OCPs 混标, 进行提取和测定, 结果见表 1,21 种 OCPs 的回收率均值范围为 $68.3 \% \sim 113.0 \%$, 相对标准偏差 (RSD) 范围为 $4.25 \% \sim 8.99 \%$. 样品检测的同 时完成方法空白, 方法空白无 OCPs 检出. 本研究数据没有用回收率校正.

表 1 千岛湖表层沉积物中有机氯农药的精密度与加标回收率 $(n=6)$

Tab. 1 Precision and recoveries of OCPs in the surface sediments of Lake Qiandao $(n=6)$

\begin{tabular}{|c|c|c|c|c|c|}
\hline 化合物 & 加标平均回收率/\% & 相对标准偏差 $\%$ \% & 化合物 & 加标平均回收率/\% & 相对标准偏差/\% \\
\hline$\alpha-\mathrm{HCH}$ & 86.1 & 6.22 & $\mathrm{p}, \mathrm{p}^{\prime}$-DDE & 113.0 & 7.55 \\
\hline 六氯苯 & 88.5 & 5.57 & $o, p^{\prime}$-DDD & 107.0 & 7.15 \\
\hline$\beta-\mathrm{HCH}$ & 87.2 & 5.35 & 狄氏剂 & 72.9 & 8.16 \\
\hline$\gamma$-HCH & 93.2 & 4.25 & 异狄氏剂 & 75.5 & 8.99 \\
\hline$\delta$-HCH & 87.5 & 5.65 & $\mathrm{p}, \mathrm{p}^{\prime}-\mathrm{DDD}$ & 91.5 & 8.31 \\
\hline 七氯 & 83.6 & 7.51 & o,p'-DDT & 81.3 & 7.88 \\
\hline 艾氏剂 & 76.5 & 5.89 & 硫丹 II & 68.3 & 8.53 \\
\hline 异艾氏剂 & 73.5 & 7.32 & $\mathrm{p}, \mathrm{p}^{\prime}$-DDT & 78.8 & 7.96 \\
\hline 环氧七氯 & 82.5 & 5.48 & 甲氧氯 & 88.5 & 7.11 \\
\hline$o, p^{\prime}-\mathrm{DDE}$ & 95.8 & 5.98 & 灭蚁灵 & 72.5 & 8.44 \\
\hline 硫丹 I & 71.5 & 7.38 & & & \\
\hline
\end{tabular}

\section{2 结果与讨论}

\section{1 千岛湖表层沉积物 OCPs 残留特征}

千岛湖各采样点位的 OCPs 含量和空间分布情况见表 2 和图 2 , 其残留特征主要表现如下:

1 ) 研究区 OCPs 残留水平较低, 以 DDTs 污染为主, 需对 DDTs 来源进行深人研究和合理控制. 由表 2 可 见,所测定的 7 个点位样品的 21 种 OCPs 中, $\delta$-HCH、o, p’-DDT、艾氏剂、异艾氏剂、环氧七氯、硫丹 I、狄氏 剂、异狄氏剂、硫丹II 等 9 种 OCPs 未检出, 其余 12 种均有不同浓度检出, $\sum$ OCPs 含量范围为 $0.43 \sim 12.70$ $\mathrm{ng} / \mathrm{g}$, 平均值为 $5.28 \pm 4.84 \mathrm{ng} / \mathrm{g}$. 所有点位均检出 DDTs, $\sum$ DDTs 约占 $\sum$ OCPs 的 $70.2 \%$, 是主要污染因子. $\sum$ HCHs 仅约占 $\sum$ OCPs 的 $3.1 \%$ ，含量远低于其他有机氯农药. 与国内外其他主要湖库表层沉积物中 OCPs 含量相比较,太湖、洪湖、鄱阳湖、北美 Ontario 湖、加拿大 Yukon 湖 ${ }^{[10-14]}$ 表层沉积物中 $\sum$ DDTs 含量均值范围 为 $1.08 \sim 141.00 \mathrm{ng} / \mathrm{g}, \sum \mathrm{HCHs}$ 含量均值范围为 $0.57 \sim 11.19 \mathrm{ng} / \mathrm{g}$, 而千岛湖表层沉积物中 $\sum$ DDTs 含量均 值为 $3.70 \pm 4.21 \mathrm{ng} / \mathrm{g}$, 相比处于低残留水平, $\sum H C H s$ 含量均值为 $0.15 \pm 0.40 \mathrm{ng} / \mathrm{g}$, 远低于其他地区.

2 ) 灭蚁灵的全区域检出需引起关注. 灭蚁灵是 POPs 公约中首批列出的 12 种最具有风险的持久性有机 污染物之一. 本研究中, 灭蚁灵在 7 个点位均有检出, 含量范围为 $0.13 \sim 0.58 \mathrm{ng} / \mathrm{g}$, 分布呈现均一性(表 2 ).

3 ) 结合表 2 、图 2 可知,西北角的街口点位 (人境断面) 和东南角的大坝前点位 (出境断面)存在 OCPs 
高残留, $\sum$ OCPs 浓度分别为 11.10 和 $12.70 \mathrm{ng} / \mathrm{g}$. 街口点位是 HCHs 、六氯苯、七氯输人的唯一点位, 浓度分 别为 $1.05 、 7.20$ 和 $0.48 \mathrm{ng} / \mathrm{g}$, 可能原因是街口点位作为安徽上游来水进人千岛湖的人境断面, 因接纳新安 江干流沿岸部分工业、农业和生活污水, 造成其污染源输人的独特性. 东南角的大坝前点位主要是 DDTs 污 染, DDTs 浓度达 $12.50 \mathrm{ng} / \mathrm{g}$, 这可能与该点位附近的人类工农业活动频繁有关.

表 2 千岛湖表层沉积物中 OCPs 的含量 $(\mathrm{ng} / \mathrm{g})$ *

Tab. 2 Concentrations of OCPs in the surface sediments of Lake Qiandao

\begin{tabular}{|c|c|c|c|c|c|c|c|c|}
\hline \multirow{2}{*}{ 化合物 } & \multicolumn{7}{|c|}{ 采样点位 } & \multirow{2}{*}{ 平均值 } \\
\hline & 街口 & 小金山 & 茅头尖 & 航头岛 & 三潭岛 & 西园库湾 & 大坝前 & \\
\hline$\alpha-\mathrm{HCH}$ & 0.04 & ND & ND & ND & ND & ND & ND & 0.01 \\
\hline$\beta-\mathrm{HCH}$ & 0.94 & ND & ND & ND & ND & ND & ND & 0.13 \\
\hline$\gamma$-HCH & 0.06 & ND & ND & ND & ND & ND & ND & 0.01 \\
\hline$\delta-\mathrm{HCH}$ & ND & ND & ND & ND & ND & ND & ND & ND \\
\hline$\sum \mathrm{HCHs}$ & 1.05 & ND & ND & ND & ND & ND & ND & 0.15 \\
\hline$o, p '$-DDE & ND & ND & 1.69 & ND & 0.64 & 0.22 & 1.90 & 0.64 \\
\hline p,p'-DDE & 0.31 & 0.22 & 1.21 & ND & 1.21 & 0.63 & 4.92 & 1.21 \\
\hline$o, p^{\prime}$-DDD & ND & ND & 0.12 & ND & 0.16 & ND & 0.73 & 0.14 \\
\hline p,p' -DDD & 0.30 & ND & 0.64 & ND & 0.85 & 2.22 & 3.22 & 1.03 \\
\hline$o, p '$-DDT & ND & ND & ND & ND & ND & ND & ND & ND \\
\hline p,p'-DDT & 1.18 & ND & ND & ND & 0.32 & 0.46 & 1.16 & 0.44 \\
\hline 甲氧氯 & ND & ND & ND & 0.15 & ND & 0.91 & 0.59 & 0.24 \\
\hline$\sum$ DDTs & 1.79 & 0.22 & 3.66 & 0.15 & 3.17 & 4.44 & 12.50 & 3.70 \\
\hline 六氯苯 & 7.20 & ND & ND & ND & ND & ND & ND & 1.03 \\
\hline 七氯 & 0.48 & ND & ND & ND & ND & ND & ND & 0.07 \\
\hline 灭蚁灵 & 0.58 & 0.21 & 0.17 & 0.45 & 0.13 & 0.51 & 0.23 & 0.32 \\
\hline$\sum$ OCPs & 11.10 & 0.43 & 3.83 & 0.60 & 3.30 & 4.95 & 12.70 & 5.28 \\
\hline
\end{tabular}

* ND 表示未检出.

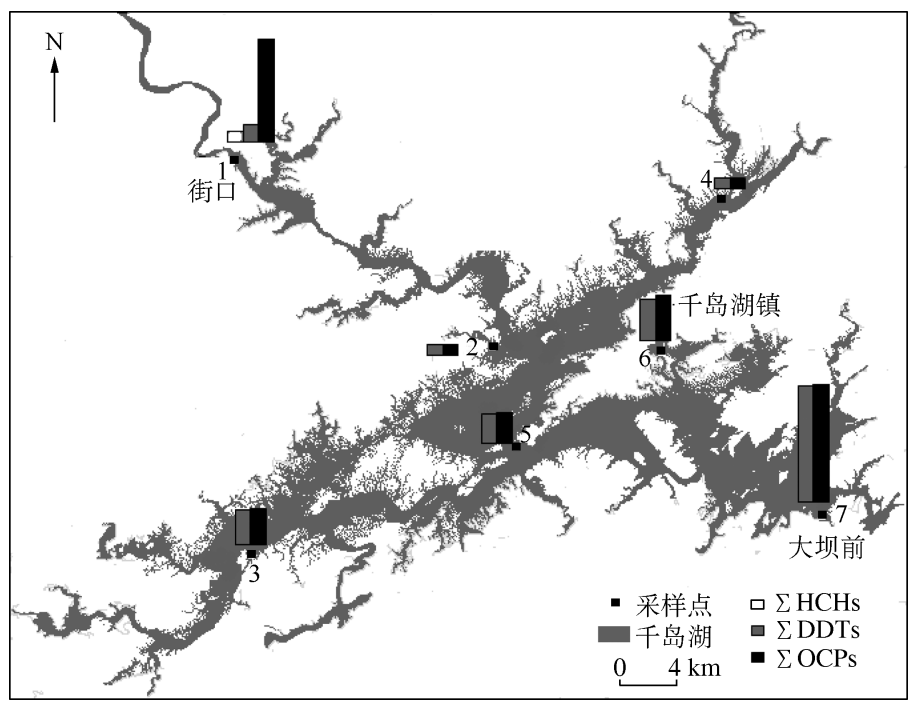

图 2 千岛湖表层沉积物中 OCPs 含量的空间分布

Fig. 2 Spatial distribution of OCPs concentrations in the surface sediments of Lake Qiandao 


\section{2 千岛湖表层沉积物 HCHs 与 DDTs 的组成特征及源解析}

2.2.1 HCHs 的组成特征及源解析 对母体化合物及其代谢产物比率的研究将有助于对新旧污染源进行判

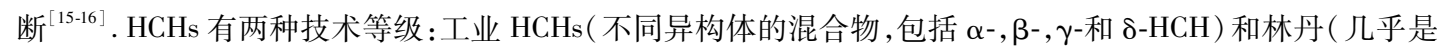
纯 $\gamma-\mathrm{HCH})^{[17]}$, 其中工业 HCHs 的使用更为广泛. 各异构体在空间构型上的差异决定了其稳定性为 $\beta$ - $\mathrm{HCH}>$ $\delta-\mathrm{HCH}>\alpha-\mathrm{HCH}>\gamma-\mathrm{HCH}$, 其中 $\alpha-\mathrm{HCH}$ 在环境中挥发性较强, $\gamma-\mathrm{HCH}$ 可以被降解 ${ }^{[18]}$, 或通过生物过程转变 成其它异构体, 而 $\beta-\mathrm{HCH}$ 的结构比其它异构体更稳定, 不容易被微生物降解 ${ }^{[19-20]}$, 能稳定存在于环境中. 一 般而言, 当沉积物中 $\alpha-\mathrm{HCH} / \gamma-\mathrm{HCH}$ 比值在 $0.2 \sim 1.0$ 之间时, 表明存在林丹的污染; 当该值在 $4 \sim 15$ 之间 时, 说明其可能来源于工业 HCHs 的输人 ${ }^{[21]}$. 千岛湖街口点位沉积物中 $\alpha-\mathrm{HCH} / \gamma-\mathrm{HCH}$ 的比值为 0.67 , 说明 该点位样品存在林丹的污染. $\beta-\mathrm{HCH}$ 约占 $\sum \mathrm{HCHs}$ 的 $90.4 \%$, 其它异构体占 $\sum \mathrm{HCHs}$ 的 $9.6 \%$, 表明该点位沉 积物中的 HCHs 已发生一定程度的降解, 近期基本无新的 HCHs 输人, 主要来源应为早期残留.

2.2.2 DDTs 的组成特征及源解析 工业 DDTs 和三氯杀螨醇是环境中 DDTs 的主要来源. 工业 DDTs 的主要 成分为 $\mathrm{p}, \mathrm{p}^{\prime}$-DDT 和少量 $\mathrm{o}, \mathrm{p}^{\prime}-$-DDT, 而三氯杀螨醇则含有 $\mathrm{p}, \mathrm{p}^{\prime}$-DDT、 $\mathrm{o}, \mathrm{p}^{\prime}$-DDT、 $\alpha$-Cl-DDT、 $\mathrm{p}, \mathrm{p}$ '-DDE 以及 $o, p^{\prime}$-DDE 等杂质 ${ }^{[22-23]}$. 此外, 由于结构原因, 在三氯杀螨醇生产过程中, $o, p^{\prime}$-DDT 杂质的含量比 $\mathrm{p}, \mathrm{p}^{\prime}$-DDT 杂质要高, 对市场上的三氯杀螨醇产品的抽样调查发现, o, p' -DDT 的含量约为 $\mathrm{p}, \mathrm{p}$ ' -DDT 的 7 倍 ${ }^{[22-23]}$. 在多 数情况下, p, p' -DDE 被认为仅来源于工业 DDTs, 因此许多研究者将 $\mathrm{p}, \mathrm{p}^{\prime}$-DDT/p, p' -DDE 的大小作为有无 新的工业 DDTs 输人的判断依据, 若比值远小于 1 , 认为是老化残留, 最近无新的工业 DDTs 的输人; 若比值 大于 1 , 则认为最近有工业 DDTs 的输人 ${ }^{[24-25]}$. 然而, 在既有工业 DDTs 施用历史也有三氯杀螨醇施用的区 域, p, p'-DDE 不仅来源于工业 DDTs 中 p,p'-DDT 的降解, 而且也来源于三氯杀螨醇的降解. 因此, 仅采用 $\mathrm{p}, \mathrm{p}^{\prime}$-DDT/p,p'-DDE 来对 DDTs 残留进行源解析是不科学的. 在本研究中, p, p'-DDT、, $\mathrm{p}^{\prime}$ '-DDE 和 p, p' -DDD 的总浓度远大于 o, p'-DDT、o, p'-DDE 和 o, p' -DDD 的总浓度, 表明 p, p' -DDT 及其衍生物是研 究区 DDTs 的主要残留形态. p, p' -DDT 是工业 DDTs 的主要成分, $o, p^{\prime}$-DDT 作为三氯杀螨醇的最主要的杂 质成分在研究区并未被检出, 可见工业 DDTs 的历史使用是千岛湖表层沉积物 DDTs 的主要来源.

确认工业 DDTs 的历史使用为主要来源后, 根据 Hong 等研究 ${ }^{[26]}$ 判断各个点位有无新的工业 DDTs 输 人. 当 (DDD $+\mathrm{DDE}) / \sum \mathrm{DDTs}>0.5$ 时, 可认为 DDT 污染的土壤 (沉积物) 经长期风化, 沉积物中 DDTs 主要 来自环境残留,而不是来自新的污染物输人; 反之, 当 $(\mathrm{DDD}+\mathrm{DDE}) / \sum \mathrm{DDTs}<0.5$ 时, 则环境中有新的 DDTs 输人. 经计算, 仅街口点位 $(\mathrm{DDD}+\mathrm{DDE}) / \sum \mathrm{DDTs}=0.34<0.5$, 表明环境中有新的 DDTs 输人, 其余 6 个点位 $(\mathrm{DDD}+\mathrm{DDE}) / \sum \mathrm{DDTs}$ 均远大于 0.5 , 表明沉积物中 DDTs 主要来自环境残留.

\section{3 千岛湖表层沉积物 OCPs 生态风险评价}

2.3 .1 沉积物质量基准法 沉积物质量基准法 ( SQGs) 是评估淡水、港湾和海洋沉积物质量的有用工具, Long 等 ${ }^{[27]}$ 在大量实验研究的基础上提出用于确定河口、海洋沉积物中有机污染物的潜在生态风险的效应 区间低值 (Effects Range Low，ERL) 和效应区间中值 (Effects Range Median，ERM), 并被视为反映沉积物质 量的生态风险水平. 当污染物浓度 $<$ ERL 时, 对生物产生的毒副作用不明显 ( 风险几率 $<10 \%$ ); 当污染物浓 度 > ERM 时, 则对生物会产生毒副作用 ( 风险几率 > 50\%), 可能会产生一定程度的负面生态效应; 当污染 物浓度在 ERL 与 ERM 之间时,生物有害效应几率介于 $10 \%$ 50\% 之间, 只会偶尔产生负面效应. 用 SQGs 评 价千岛湖表层沉积物中 OCPs 的生物毒性效应见表 3, 其中列出本研究存在超标的 OCPs 的 ERL 和 ERM 值. 7 个点位中均有 5 个点位样品的 DDD、DDT 和 DDE 低于 ERL 值, 有 3 个点位样品 $\sum$ DDTs 低于 ERL 值, 茅头 尖与大坝前样品的 DDE、西园库湾与大坝前样品的 DDD、街口与大坝前样品的 DDT 以及茅头尖、三潭岛、西 园库湾与大坝前样品的 $\Sigma$ DDTs 超过了 ERL 值, 但都低于 ERM 值, 无点位样品的 OCPs 超过 ERM 值. 街口、 茅头尖、三潭岛、西园库湾与大坝前等 5 个点位样品的 OCPs 介于 ERL 值与 ERM 值之间,生物有害效应几率 介于 $10 \% \sim 50 \%$ 之间, OCPs 对该区生物可能存在生态风险.

2.3.2 沉积物质量标准法 采用加拿大魁北克省 2006 年颁布的沉积物质量标准 ( SQSs) ${ }^{[28]}$ 对千岛湖开展评 价, 该标准包含 5 个阈值, 分别为生物毒性影响的罕见效应浓度值 (REL)、临界效应浓度值( TEL)、偶然效应 浓度值( OEL)、可能效应浓度值 (PEL) 和频繁效应浓度值 (FEL), DDE、DDD、DDT、狄氏剂、异狄氏剂、环氧 七氯、 $\gamma-\mathrm{HCH}$ 等 7 种 OCPs 的上述 5 个國值可参见加拿大魁北克省 2006 年颁布的沉积物质量标准 ${ }^{[28]}$. 用这 
些阈值来评价千岛湖表层沉积物中 OCPs 的污染程度, 结果表明小金山和航头岛 7 种 OCPs 含量均小于 REL, 对底栖生物不存在生态风险; 街口和西园库湾至少有一种 OCPs 含量介于 REL 和 TEL 之间, 对底栖生 物的不良影响概率属于罕见水平; 三潭岛 DDE 含量介于 TEL 和 OEL 之间, 对底栖生物的最大不良影响概率 局限于偶然水平; 茅头尖 DDE 含量介于 OEL 和 PEL 之间, 对底栖生物可能存在生态风险; 大坝前 DDE 含量 介于 PEL 和 FEL 之间, 对底栖生物存在一定的生态风险, 需加强局部监测; 暂无点位 OCPs 含量大于 FEL 值,表明千岛湖尚不需要开展沉积物 OCPs 污染修复.

表 3 千岛湖表层沉积物中 OCPs 的质量基准评价

Tab. 3 Assessment according the quality guidelines to OCPs in the surface sediments of Lake Qiandao

\begin{tabular}{ccccccc}
\hline 化合物 & $\mathrm{ERL} /(\mathrm{ng} / \mathrm{g})$ & $\mathrm{ERM} /(\mathrm{ng} / \mathrm{g})$ & 本研究/ $(\mathrm{ng} / \mathrm{g})$ & $<\mathrm{ERL}$ 的点位 介于 $\mathrm{ERL}$ 与 ERM 之间的点位 & $>\mathrm{ERM}$ 的点位 \\
\hline DDE & 2.2 & 7.0 & $0 \sim 6.82$ & $1 、 2 、 4 、 5 、 6$ & $3 、 7$ & - \\
DDD & 2.0 & 20.0 & $0 \sim 3.95$ & $1,2 、 3 、 4 、 5$ & $6 、 7$ & - \\
DDT & 1.0 & 27.0 & $0 \sim 1.18$ & $2 、 3 、 4 、 5 、 6$ & 1,7 & - \\
$\sum$ DDTs & 3.0 & 46.1 & $0.15 \sim 12.50$ & $1,2 、 4$ & $3 、 5 、 6 、 7$ & - \\
\hline
\end{tabular}

\section{3 结论}

1) 研究区 OCPs 残留水平较低, 以 DDTs 污染为主; 街口 (人境断面)、大坝前 (出境断面) 出现 OCPs 高 残留, 街口点位是 HCHs、六氯苯、七氯输人的唯一点位, 大坝前点位样品主要受 DDTs 污染, 灭蚁灵的全区域 检出需引起关注.

2) HCHs 的组成特征及源解析表明仅街口点位存在林丹的污染; DDTs 的组成特征及源解析则表明工 业 DDTs 的历史使用是千岛湖表层沉积物 DDTs 的主要来源,仅街口点位有新的 DDTs 输人.

3) 利用沉积物质量基准法、沉积物质量标准法分别对千岛湖表层沉积物中 OCPs 的生态风险进行评 价,结果表明部分点位如大坝前、茅头尖样品中 OCPs 的残留现状对该区生物可能存在生态风险,需加强局 部监测.

\section{4 参考文献}

[ 1 ] Rodan BD, Pennington DW, Eckley N et al. Screening for persistent organic pollutants: techniques to provide a scientific basis for POPs criteria in international negotiations. Environmental Science and Technology, 1999, 33 : 3482-3488.

[ 2 ] Voldner EC, Li YF. Global usage of selected persistent organochlorines. Science of the Total Environment, 1995, 160/ 161 : 201-210.

[ 3 ] Hua XM, Shan ZJ. The production and application of pesticides and factor analysis of their pollution in environment in China. Advance of Environmental Science, 1996, 2 : 33-45.

[ 4 ] Hu GC, Luo XJ, Li FC et al. Organochlorine compounds an d polycyclic aromatic hydrocarbons in surface sediment from Baiyangdian Lake, North China: Concentrations, sources profiles and potential risk. Journal of Environmental Sciences, $2010,22(2): 176-183$.

[ 5] 黎 冰, 解启来, 廖 天等. 扎龙湿地表层沉积物有机氯农药的污染特征及风险评价. 农业环境科学学报,2013,32 (2) : 347-353.

[ 6 ] 余员龙,任丽萍,刘其根等. 2007-2008 年千岛湖营养盐时空分布及其影响因素. 湖泊科学,2010,22(5):684-692.

[ 7 ] 李培培, 史 文, 刘其根等. 千岛湖叶绿素 a 的时空分布及其与影响因子的相关分析. 湖泊科学, 2011,23(4): 568-574.

[ 8 ] 高玉蓉, 刘明亮, 吴志旭等. 应用实测光谱估算千岛湖夏季叶绿素 a 浓度. 湖泊科学, 2012,24(4):553-561.

[9] 李共国,虞左明. 千岛湖轮虫群落结构及水质生态学评价. 湖泊科学, 2003,15(2): 170-176.

[10］袁旭音, 王 禹, 陈 俊等. 太湖沉积物中有机氯农药的残留特征及风险评估. 环境科学, 2003,24(1):121-125.

[11] 龚香宜, 祁士华, 吕春玲等. 洪湖表层沉积物中有机氯农药的含量及组成. 中国环境科学, 2009,29(3):269-273.

[12] 黄 云,钟恢明,刘志刚. 鄱阳湖沉积物中主要有机氯农药的残留特征. 江西科学,2010,28(3):336-340. 
[13] Oliver BG, Niimi AJ. Trophodynamic analysis of polychlorinated biphenyl congeners and other chlorinated hydrocarbons in the lake Ontario ecosystem. Environmental Science and Technology, 1988, 22 :388-397.

[14] Rawn DFK, Laekhart WL, Wilkinson P. Historical contamination of Yukon Lake sediments by PCBs and organochlorine pesticides: Influenee of local sources and watershed characteristics. Science of the Total Environment, 2001, 280 : 17-37.

[15 ] Harner T, Wideman JL, Jantunen LMM et al. Residues of organochlorine pesticides in Alabama soils. Environmental Pollution, 1999, 106: 323-332.

[16] Tavares TM, Beretta M, Costa MC. Ratio of DDT/DDE in the all saints, Brazil and its use in environmental management. Chemosphere, 1999, 38(6): 1445-1452.

[17] Li YF, Cai DJ, Singh A. Technical hexachlorocyclohexane use trends in China and their impact on the environment. Archives of Environmental Contamination Toxicology, 1998, 35: 688-697.

[18] Kaushik CP. Persistence and metabolism of HCH and DDT under subtropical conditions. Soil and Biology and Biochemistry, 1991, 23(2) : 131-134.

[19] Kalbirz K, Popp P, Geyer W et al. $\beta-\mathrm{HCH}$ mobilization in polluted wetland soils as influenced by dissolved organic matter. Science of the Total Environment, 1997, 204(1) : 37-48.

[20] Middeldorp PJM, Jaspers M, Zehnder AJB et al. Biotransformation of $\alpha-, \beta-, \gamma^{-}$, and $\delta$-hexachlorocyclohexane under methanogenic conditions. Environmental Science and Technology, 1996, 30(7) : 2345-2349.

[21] Kalantzi OI, Alcock RE, Johnston PA et al. The global distribution of PCBs and organochlorine pesticides in butter. Environmental Science and Technology, 2001, 35(6) : 1013-1018.

[22] Qiu XH, Zhu T, Yao B et al. Contribution of dicofol to the current DDT pollution in China. Environmental Science and Technology, 2005, 39: 4385-4390.

[23] Qiu XH, Zhu T, Jing L et al. Organochlorine pesticides in the air around the Taihu Lake, China. Environmental Science and Technology, 2004, 38: 1368-1374.

[24] Aigner EJ, Leone AD, Falconer RL. Concentrations and enantiomeric ratios of organochlorine pesticides in soils from the U. S. Corn Belt. Environmental Science and Technology, 1998, 32 : 1162-1168.

[25] Dimond JB, Owen RB. Long-term residue of DDT compounds in forest soils in Maine. Environmental Pollution, 1996, 92 : 227-230.

[26] Hong H, Chen W, Xu L et al. Distribution and fate of organochlorine pollutants in the Pearl River Estuary. Marine Pollution Bulletin, 1999, 39: 376-382.

[27] Long ER, Macdonald DD, Smith SL et al. Incidence of adverse biological effects with range of chemical concentrations in marine and estuarine sediments. Environmental Management, 1995, 19(1) : 81-97.

[28 ] Environment Canada and Ministère du développement durable, de I' Environment et des Parcs du Québec. Criteria for the assessment of sediment quality in Quebec and application frameworks : prevention, dredging and remediation. Québec: Library and Archives Canada Cataloguing in Publication, 2007: 1-39. 